\title{
Beyond Educational Videogames to Educational Systems-That-Incorporate Videogames: A Case Study of a System for Learning about Energy
}

\author{
Nick V. Flor, Rodrigo Arias, Meghana Dasigi, Megan Hayden, Melinda Mesibov, Keara Sweeney \\ University of New Mexico \\ nickflor@unm.edu
}

\begin{abstract}
A common goal for designers of educational videogames is to make learning fun. Unfortunately, the result is often a game that tries to combine the fun aspects of videogames with learning elements, but that is neither fun nor effective for learning. In this paper we present our discovery of an alternative approacha system that combines both education and entertainment, but that separates them into different modules that are loosely-coupled. Entertainment motivates education through a reward mechanism, where performance in the education module yields tokens that can be redeemed for in-game assets in the entertainment module. We present a case study of our specific implementation of this system, and we discuss how it can be generalized to motivate the learning of any topic where performance can be measured. This research contributes to our understanding of designing cognitive artifacts, and to our understanding of designing educational systems as distributed services.
\end{abstract}

\section{Introduction}

The primary goal of educational videogame designers is to create games that make learning fun. But often the result is neither as entertaining nor as educational as it could have been, had the designer focused exclusively on creating a fun game or on simply placing the educational information online - a tradeoff exists between entertainment value and educational content.

This tradeoff between entertainment and education will always be present in some measure because learning styles differ. For example, take the task of learning the multiplication table. A game can certainly make the task more fun by adding appealing graphics and attention-grabbing animations (e.g., Math Rabbit by The Learning Company), but fundamentally it is a memorization task. Learners who are able to focus on memorizing a table of numbers will find playing the game less effective than rehearsing the table.

We are not arguing against creating educational videogames, just that it is non-trivial to blend education and entertainment. Numerous factors make the blending difficult, which include the learning topic, learning styles, and even the learner's culture. If a game does not account for these factors, it may be fun but not an effective learning tool in general. Is there an alternative approach - an artifact that can maximize both entertainment and education?

In this paper we present a case study of one such alternative approach. Rather than designing educational video games, per se, we argue for designing educational systems-that-incorporate videogames. Our approach separates learning and fun into different "modules" so that: (a) teachers can focus on providing content that maximizes learning for different learner styles, and (b) video game designers can concentrate on making a variety of games that are as fun as possible without worrying about adding learning content. The modules are related by a reward system, where a learner's performance in the education module yields merit tokens ("Meritokens") that learners can redeem for in-game assets in the entertainment module. In this way games motivate learning without having to mesh learning.

\section{Background: Virtual worlds, service science, and distributed cognition}

There are many definitions of virtual worlds [1,2], but when referring to a 3-D virtual world it is: a computer-mediated interactive space, where the objects in the world are depicted with depth, and where the objects behave according to specific rules as programmed by the software architect(s). Users interact with these objects via an avatar, and there may be multiple userssimultaneously within the virtual world. The objects depicted in a 3-D virtual world can range from abstract [3] to realistic [4]. 
Virtual worlds provide flexible and controlled environments for the scientific study of social, behavioral, business, and economic phenomenon $[5$, 6]. But they are also a promising environment for teaching and learning $[7,8]$.

There are numerous design guidelines for virtual worlds, which focus on what designers should include within a virtual world $[9,10]$. However, what the current virtual world design research does not address is how to design learning systems, where the virtual world is not the primary service, but one part of a larger educational service for accomplishing some learning outcome.

Service science provides a framework for thinking about the embedding of virtual worlds as one component in a larger service system.

Service science is the study of service systems [11], where a service system is a value co-creating organization of: (a) people; (b) technology; (c) value propositions connecting internal and external elements; and (d) shared information. The smallest service system is an individual interacting with other people and tools to accomplish work, and the largest service consists of nations interacting with one another, mediated by technologies, contracts, tariffs and other macro-level rules and regulation. A virtual world can be either the service system itself or a component of a larger service system.

A possible philosophical foundation for service science is service-dominant logic ("S-D Logic") [12], which is a world view encompassed by 10 foundational principles [13]: (1) service is the fundamental basis of exchange; (2) indirect exchange masks the fundamental basis of exchange; (3) goods are distribution mechanisms for service provision; (4) operant resources are the fundamental source of competitive advantage; (5) all economies are service economies; (6) the customer is always a co-creator of value; (7) the enterprise cannot deliver value, but only offer value propositions; (8) a service-oriented view is inherently customer oriented and relational; (9) all economic and social actors are resource integrators; (10) value is always uniquely and phenomenologically determined by the beneficiary.

Service science and S-D logic, provide designers a way of thinking about a virtual world in terms of systems of agents, services provided, value co-created, artifacts mediating services, and efficient distributions of work among agents based on value propositions. The virtual world alone is not necessarily the primary service system, but a component that offers a very specific value proposition, which includes immersion, interactivity, and simulation.

But service science can benefit from other logics, particular when designers are creating the lowest-level of service system consisting of individual users interacting with technologies. At this level the problem consists typically of designing new representations or finding effective distributions of cognitive abilities across people and tools.

The theoretical framework of distributed cognition $[14,15]$, with its emphasis on artifacts, representations, and processes, provides a logic for designing the details of service systems at the individual-technology level. It is the key theoretical framework underlying our research and informing our design decisions.

A key axiom of the distributed cognition framework is that cognitive structures and processes that have been traditionally located inside the heads of individuals - such as motivation, attention, perception, decision making, and memory - are, in fact, general structures and processes found in any system that retrieves, processes, stores, and forwards information. So, for example, take a human-machine information system consisting of a person, a computer, and post-it notes. If the person places a post-it note, with a handwritten reminder, in the middle of his or her computer screen, that artifact is a kind of extrinsic attention and memory device [16].

One can therefore use the language of informationprocessing psychology to describe any observable information system, such as human-machine information systems. The process of describing a system in this manner is known as cognitive ethnography, and an important outcome of a cognitive ethnography is a map of the cognitive ecology - the structures and processes employed as the system operates.

For human-computer interaction designers, this cognitive map can serve as the basis for designing new systems that improve or extend the capabilities of existing systems. For example, take the post-it-note qua extrinsic attention \& memory. One can ask whether there is a better design, such as using a custom screen saver as attention or memory, instead of the post-it note.

The key question when designing a new system using the distributed cognition framework becomes-is there a better representation or distribution of these cognitive structures \& processes: within individuals, across individuals \& artifacts, or implemented as artifacts?

The distributed cognition logic (or " $\mathrm{D}-\mathrm{C}$ Logic") is summarized in two principles [17]: (1) The unit of analysis is determined by the elements that participate in the process of interest and that have a functional relationship to one another. In the post-it note example, this is the person, computer and post-it note as elements of a reminder process; and (2) the range of mechanisms that participate in cognitive processes are 
flexible and do not have to reside solely in the skulls of individual actors. The process can incorporate both actor internal and actor external structures, as in the post-it note example.

\section{Method}

Two methods were used in this research: rapid prototyping [18] and the case study method [19].

We used rapid prototyping to develop several iterations of the 3D Virtual Energy World. Rapid prototyping combines software development with feedback from end-user focus groups. Feedback is used by the development teams to either incrementally improve or drastically change the software. The process of development and feedback continues until the user is satisfied with the software.

We used the case study method to build theories from our data, and to connect our data with theoretical constructs from both service science and distributed cognition. This is accomplished by comparing an early, unsatisfactory prototype with a later prototype and analyzing whether the changes follow from either service science theory or distributed cognition.

\section{Case study: 3D Virtual Energy World, an initial attempt at an educational videogame}

In 2012 the University of New Mexico was awarded a large, 4-year grant from the National Science Foundation to develop renewable energy technologies and to educate the public on the benefits of alternative energy sources. The grant was multidisciplinary, with the overall research group consisting of investigators from the schools of Engineering, Fine Arts, and Business Administration.

Our specific research team was tasked with developing educational materials about the costs and benefits of alternative energy sources, targeted at high school students through college freshmen. The team consisted of a principal investigator, a graduate research assistant, and several undergraduate research assistants majoring in either digital media or business. The team operated very similar to an advertising agency with the principal investigator serving as a creative director, the graduate student doing research, and the undergraduate students serving as the creative team, both pitching creative designs and implementing those designs.

The main challenge for our research team was developing educational materials for technologies either that did not yet exist — such as energy harvesters, which converted not only sunlight into electricity, but also moonlight, movement, heat, and electromagnetic (radio) waves - or that existed but were not commercially available. We adopted an iterative approach based on rapid prototyping.

\subsection{Focus group: Educators}

To help meet this challenge, we conducted a focus group with five science \& math teachers spanning elementary, middle, and high school. Two points of consensus emerged from this focus group. The first point was that it was crucial to educate students as early as possible on the benefits of renewable and sustainable energy. As one teacher put it:

"... biggest point is getting them while they are young. You know once you are getting them to upper high school, they're kinda already starting to get set in their ways and, you know, their family the way they've always done it and the way they're always gonna do it. I think that's the only way you're ever going to have change."

The second point of consensus was that for broad accessibility, it should be some kind of app:

"... the beauty of the apps is like our kids are very low income. A lot of them don't have computers at home, but you can bet that if they don't have a computer, they're gonna have a smart phone."

There was no consensus on the best way to present the information as an app, only that the information be understandable by students with a semester of basic science and that the app provide an enjoyable learning experience. However, a majority remarked that showing students how much energy was wasted could be an effective strategy:

"One thing I try to look at with students is teaching this idea of waste and how to conserve things"

\subsection{Response: First implementation of the 3D Virtual Energy World}

Based on the results of the focus group it became apparent that prior to educating students on the costs and benefits of alternative energy sources, they needed to be educated on energy conservation which in turn required learning: (a) appliance energy requirements; and (b) the costs of running appliances over a specific 
time period. Our research team determined that the best technology for educating students on energy conservation and alternative energy was an immersive 3D Virtual Energy World, where students would be given a house, and they would have to efficiently manage the energy consumption in that house. Figure 1 depicts a typical avatar and home in the 3D Virtual Energy World.

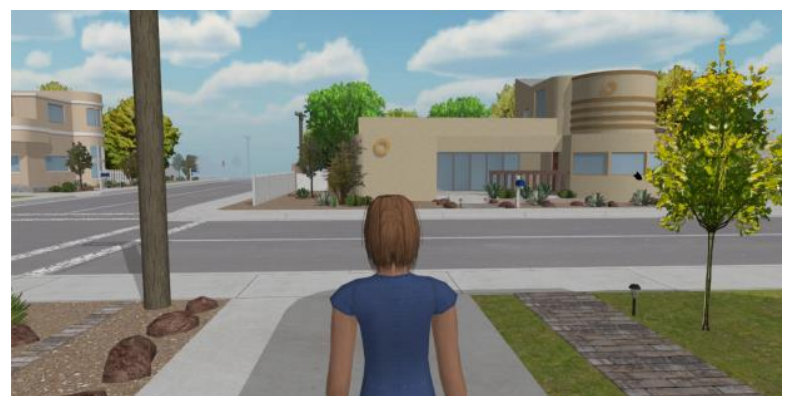

Figure 1. An avatar and house in the 3D Virtual Energy World

The houses would initially run on electricity provided by a fictitious electric company, but through efficient energy management, users could save up money to purchase renewable energy technologies like solar panels, wind mills, or energy harvesters, and consequently experience the costs and benefits of these renewable energy sources.

Each house contained the most common household utilities, such as lights, kitchen appliances, stereos, and computers. Appliances outlined in red were off, and those outline in green were on. By hovering over an appliance, its wattage would appear. For example in Figure 2, the 900 over the coffee maker indicates that it uses 900 watts. The total wattage of all "on" appliances is displayed in the upper right corner.

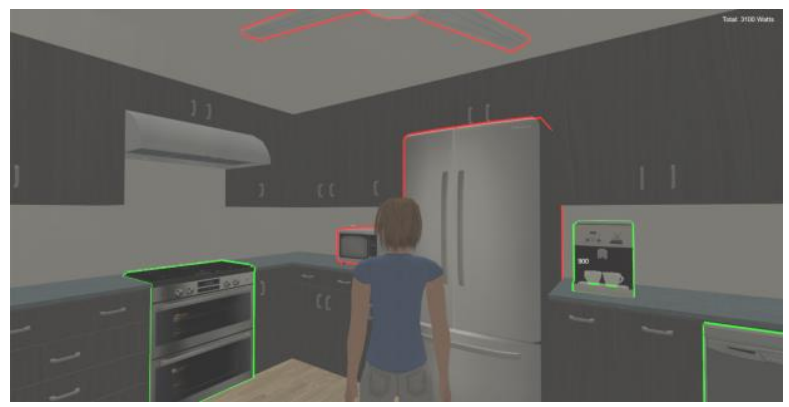

Figure 2. The interior of a house in the 3D Virtual Energy World

The 3D Virtual Energy World also gave users the ability to forecast energy usage and the costs of energy usage over time, by entering the number of hours the appliance would be on per day (see Figure 3).

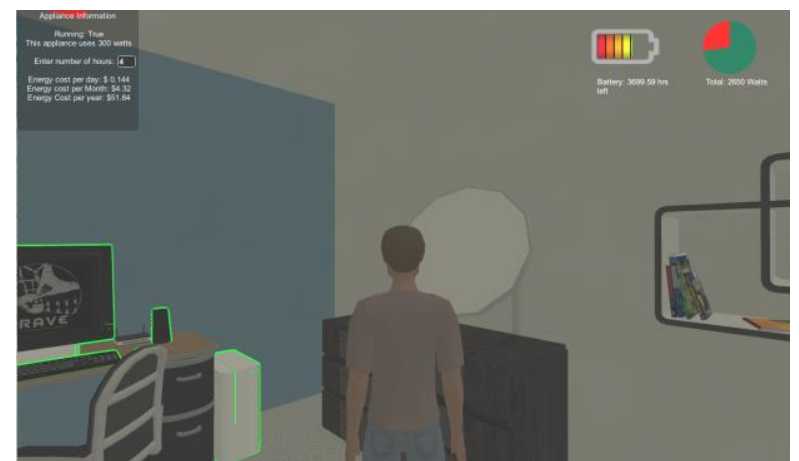

Figure 3. Forecasting energy usage and costs in the 3D Virtual Energy World

The high-level design of our system is captured by the dataflow diagram in Figure 4, which depicts most educational video games. Specifically, an educational video game developer creates a game containing educational content, and where student learning performance (e.g., memorizing appliance wattages and conserving energy) allows them to purchase in-game items (e.g., solar panels, that improve gameplay).

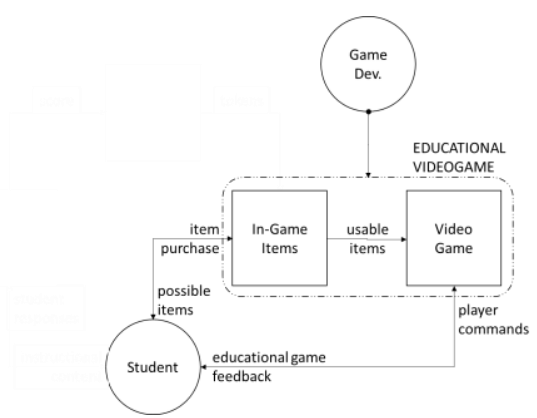

Figure 4. General high-level design of an educational videogame

\subsection{Feedback: Gamers}

Satisfied with the initial implementation and the educational aspects of the 3D virtual simulation, we decided to do another focus group, this time with 15 undergraduate and graduate students in the university's videogame club. The feedback from gamers was polite but universally negative. The consensus was that it looked nice, that they could see it working as an educational game, but that it was not fun. They would not play it unless they were forced to do so. As one gamer put it:

"Okay I don't know anything about electricity so I can see it being fun for a little bit but I wouldn't play it unless it was part of a class and I was forced to." 
When asked how to improve it the consensus was there had to be a compelling need for users to learn about electricity and to conserve it. As another gamer commented:

"You can make it like the movie Omega man where if they don't save enough electricity to power the electric fences zombies will invade your home in the night and kill you...that would make it fun."

Thus, like many educational videogame designers, we had created software that could be used for learning, but that was not fun and would not be played by gamers unless it was part of a class activity.

\subsection{Response: Design team}

In an attempt to add fun to the 3D Virtual Energy World fun, the creative team first wrote a backstory for what they believed would be an entertaining game:

"The kid arrives into town to visit his uncle and slowly starts noticing really weird stuff, people are so sick that they are not zombies but act like them, everything is dark, there is trash everywhere, etc. After, noticing that the people and the town are like that because they did not care about energy. All the quests that save energy help the town get better, but while working on them he finds out that there is a Smog Worm that was born because that town did not care about energy, he feeds of all the smog and misuse of energy. You are trying to stop him because he started there but then he is going to grow and go to other cities, and eventually destroy the world. The kid continues to have energy quests (they will learn about energy this way) that will save the town and eventually kill the Smog Worm. After defeating him the kid becomes famous and all the people in the world are aware that they have to use energy efficiently because that could happen again."

However, the principal investigator rejected this new direction as too far removed from the research goal of educating the public on renewable and sustainable energy. The design team was directed by the principal investigator to: "go back to the drawing board and come up with something respectable that doesn't involve zombies, worms, or other silly things".

\section{Radical redesign: The Energy Surge System-An educational system- incorporating-a videogame}

The creative team met for a brainstorming session, which initially focused on how to make the game fun but not silly. However, the team eventually started questioning whether it was even possible to make an educational game focused on energy awareness that was fun without being silly. This line of questioning ultimately lead to asking "why even make educational videogames?"

And the answer was that in a virtual world it is possible to create situations that are impossible in real world, and these unrealistic situations provide the motivation for players to learn topics that in reality have low priority - such as knowledge of appliances wattages or the hourly costs of running appliances. In short, educational videogames present situations that motivate the learning of low-priority topics.

As suggested by the gamer focus group, horror, science fiction, or dystopian environments provide familiar and proven entertaining contexts from which such situations can be designed.

The key insight was that the fun aspect of videogames provided the motivation for learning. This insight lead the creative team to explore the design of a system where entertainment and education were not contained in a single module - as they were in an educational video game - but decoupled into separate modules. "The Energy Surge System" was the result (refer to Figure 5, the Energy Robots videogame is the entertainment module).

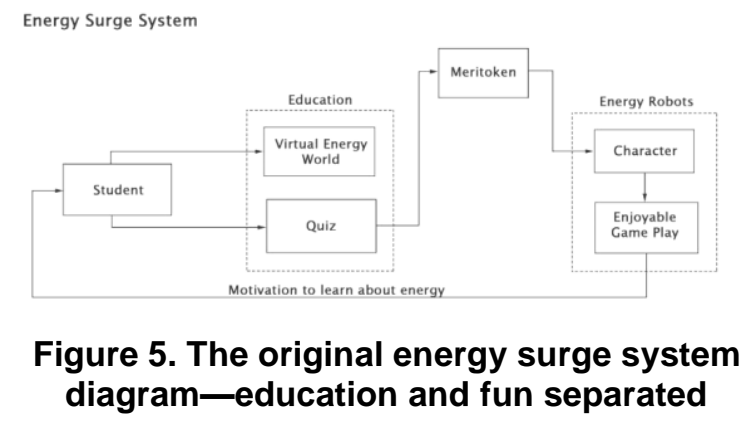

The decoupling allows an educator to use whatever materials he or she believes is best for learning without having to worry about making the learning fun. It also allows a game developer to create a game that is as fun as possible without having to worry about integrating learning content.

The videogame still motivates learning, although indirectly through a third "Meritoken" module. Specifically, students are motivated to learn because 
increased performance in the education module yields more tokens that they can redeem for in-game assets in the entertainment module, such as power-ups and new characters.

\subsection{Implementation: Education module, Virtual Energy World and quiz}

As mentioned, the learning materials in the education module can be whatever the teacher wants to use, e.g., a simple list of appliance and wattages along with a formula for converting wattages to costs. However, since it already existed, the creative team decided to reuse the Virtual Energy World as one of two instructional materials for learning about appliance wattages and costs (see Figure 6).

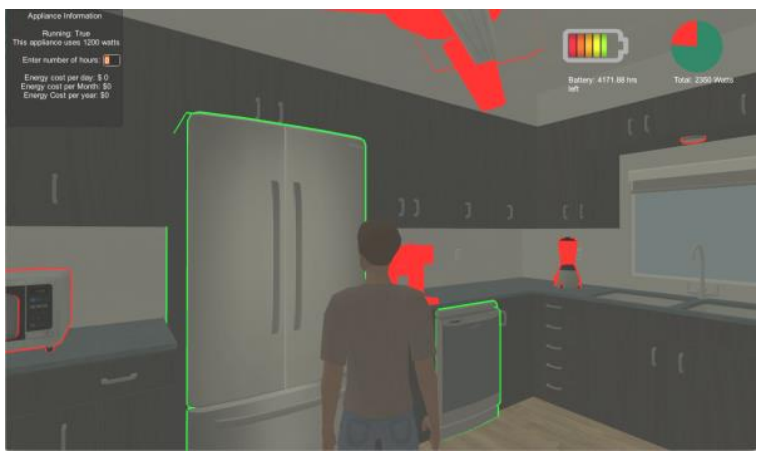

Figure 6. The Virtual Energy World reused as one part of the education module

The second instructional piece was an automated quiz that randomly asked the user energy questions related to the appliances in the Virtual Energy World (see Figure 7). The quiz was chosen because it is the most common device used by teachers to test learning.

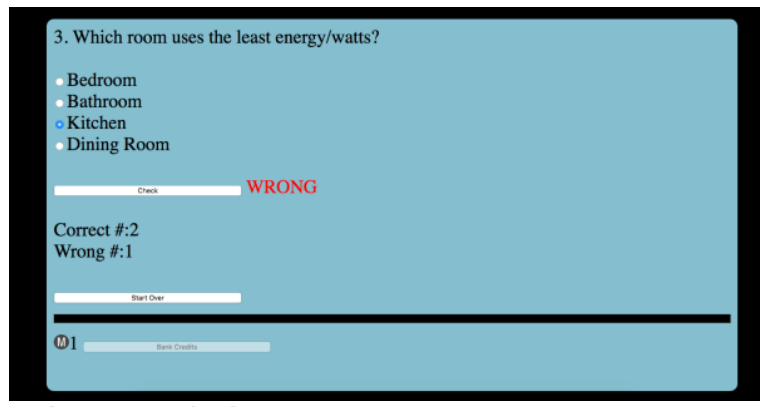

Figure 7. Quiz on energy use as the second and final piece of the education module

The automated quiz kept track of the number of correct and incorrect answers, and the difference was fed into the Meritoken Module.

\subsection{Implementation: Meritoken module \& connection to education module}

The Meritoken Module was implemented as a cloud-based system consisting of a database and a Web-API for storing and retrieving tokens based on a unique student ID, which students received by registering for the Energy Surge System.

Teachers could access the Meritoken Module in two ways. The first was manually, via a web page where the teacher could type in the number of tokens a student earned. This access method is appropriate when the education module consists of non-electronic learning materials, e.g., paper readings and paper quizzes.

The second and preferred access method for the Meritoken Module is electronic, via the Web API. For example, after students complete the energy quiz (refer to Figure 7), they can click on the Bank Credits button which automatically transfers the tokens to the database via the Web API.

\subsection{Implementation: Entertainment module, The Energy Robots Game}

The creative team set a goal of developing a game that was as fun as possible without having to worry about educational content, and that could be developed in a semester. They decided on Energy Robots (see Figure 8), a version of the classic ' 80 s arcade game Robotron 2084, but using 3D characters and robots instead of pixel art.

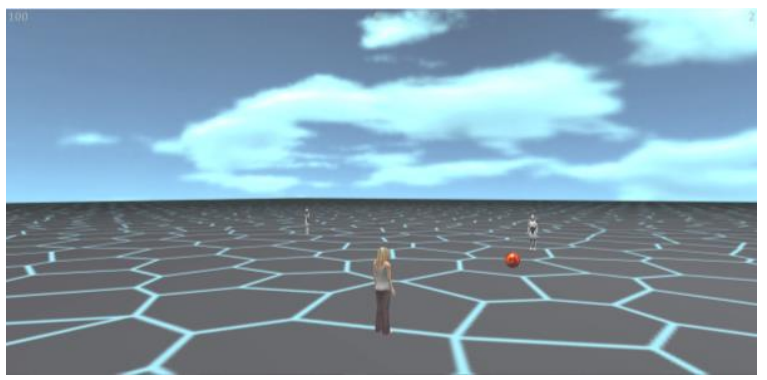

Figure 8. The Energy Robots game

Briefly, a player must shoot energy balls and destroy robots on a flat playing field. The game starts off with two robots, but continually doubles the number of robots once the player eliminates them from the playing field. 


\subsection{Implementation: Connection between the Meritoken module \& education module}

Before playing the game, users are given the option of redeeming Meritokens for characters or power-up abilities. Figure 9 depicts the interface for redeeming characters. In this particular example, the character costs 15 Meritokens to unlock, and the player has a current balance of 169 Meritokens.

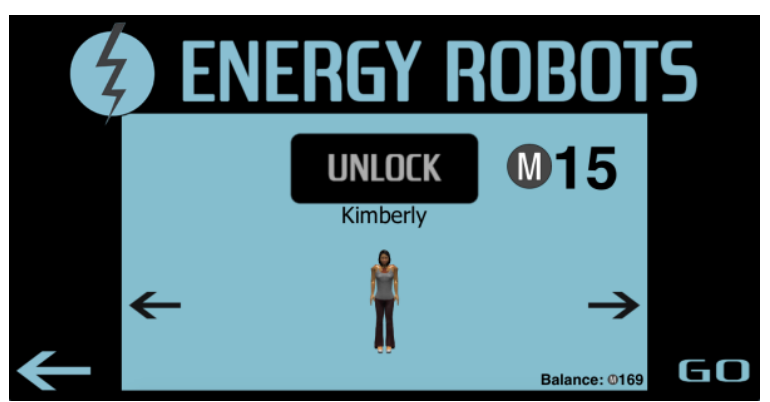

Figure 9. Redeeming meritokens for characters

\subsection{Focus group: College-level educators}

With a working prototype of the Energy Surge System, the research team conducted a focus group with six college-level educators consisting of four graduate students, a lecturer, and an assistant professor. This time the feedback was largely positive, and split between two kinds of responses. The first type, praised our use of videogames to educate students about both alternative energy and energy conservation. The following was a typical response:

"I think it was better than traditional educational video games. A really neat concept because... it teaches people about the conservation of energy. To make young people aware of the energy that they are using is a pretty prolific thing."

The second kind of response was specific to the implementation of the system, as exemplified by the following quote:

"I felt the system was really cool. I enjoyed how you started off with the educational part that was still fun to interact with. And then you gave incentives so that the user would learn as much as they can so that they can play the game after. The educational system could be a lot better to get the kids excited about things about energy or other applications. I would play this game.”

\section{Discussion}

The Energy Surge System (Figure 5) represents the smallest kind of service system: the individual-level service system. This system, in turn, embeds two other individual-level service systems - one specialized for providing education, and the other specialized for providing entertainment. The two embedded service systems are linked loosely via a third system, the Meritoken system, in which performance in the educational module provides tokens that can be redeemed for capabilities in the entertainment module.

This system evolved from negative user feedback on an earlier system - the 3D Virtual Energy Worldthat attempted to combine both education and entertainment into a single software module.

One way to use this case study to validate or refine the theories of either service science or distributed cognition, is to ask the question: is the change from one system (Virtual Energy World) to the next (Energy Surge System) prescribed by the key principles of the theories. For both theories the answer is yes in the general, but no in the specifics.

For example the very definition of a service system suggests that given an underperforming system, like the Virtual Energy World, one should attempt to find a new value co-creating configuration of people, technology, value propositions connecting internal \& external service systems, and shared information [11, p.18].

The education and entertainment modules in the Energy Surge System are two such value co-creating configurations. Furthermore, maximizing learning in the education module and fun in the entertainment module correspond to the service science aim of raising "... the competence of the provider side of service systems. Increasing competence is the key to improvement and innovation." [11, p. 19].

What does not follow clearly from the definition of a service system or from S-D logic is the Meritoken module in the Energy Surge System. Recall that performance in the educational module yields tokens that users can redeem for capabilities in the entertainment module, via the Meritoken module. In short, users are motivated to learn because they receive tokens they can redeem to increase their fun. The Meritoken module potentially maps onto either a "value proposition connecting...service systems" per the definition of a service system, or a combination of S-D Logic foundation principle 2 ("indirect exchange") and foundational principle 3 ("distribution mechanism for service provision"). The point, however, is that the mapping is unclear.

Service science theory should be refined to clarify how a service-system-linking artifact like the 
Meritoken module fits in. Alternatively, service science can incorporate other logics, like distributed cognition logic. This is especially useful at the level of the individual service system, where the competencies that must be distributed are often cognitive mechanisms.

In particular, motivation is a cognitive mechanism. Distributed cognition principle 2, states that the range for any cognitive mechanism is flexible and can extend beyond an individual's head to include a variety of material resources. Thus, if intrinsic motivation is a problem (Virtual Energy World), distributed cognition logic suggests finding an extrinsic distribution of motivation across other people and technologies. The Meritoken module - as part of a distributed motivation mechanism-follows naturally from this principle.

There are three other findings from this research that are not necessarily tied to either service science or distributed cognition. The first, and most direct, is a general purpose system for using videogames to motivate any kind of learning - an educational system that incorporates videogames as opposed to an educational videogame. Figure 10 abstracts the general diagram for such a system based on the Energy Surge System in Figure 5.

In principle this system should work with any: (a) any topic whose materials can be placed online and where learning is scored online; and (b) any videogame that has internet access. The only requirement is that the instructional and videogame modules follow the Meritoken Web API for storing and retrieving tokens.

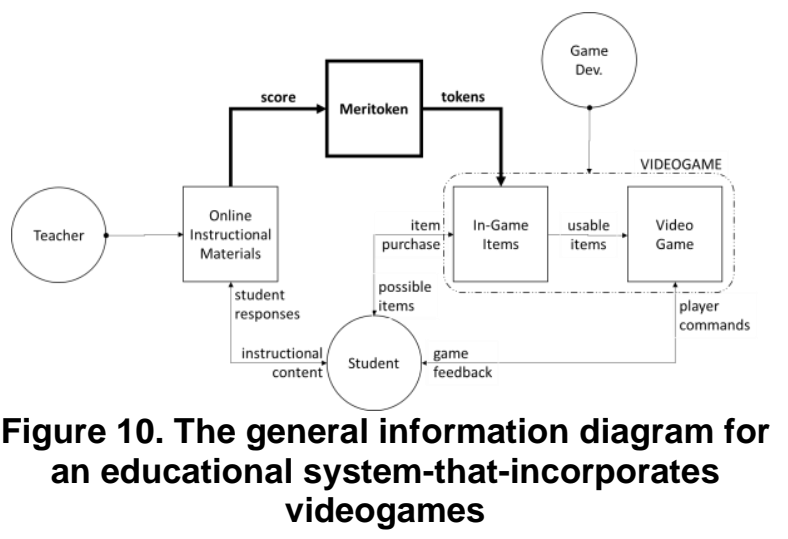

The second, is validation of the software design principle of high cohesion and loose coupling between modules [20], as applied to socio-technical systems. Unlike software, where coupling occurs through interfaces, coupling in sociotechnical systems is accomplished by a third module that relates information used in one module to that in another. Future research should explore modules beyond education and entertainment, coupling effectiveness, and different coupling mechanisms.

Third, the case study verifies Simon's observation that: "search guided by the most general heuristic of 'interestingess' or novelty is a fully realizable activity. This kind of search, which provides the mechanism for scientific discovery, may also provide the most suitable model of the social design process" [21].

This is an important observation. The emerging field of design science [22] has adopted an approach to design, where only artifacts designed by a rigorous confirmatory process are deemed scientific. However, this research demonstrates that novel artifacts can be designed through an iterative and exploratory process, guided by principles such as novelty, or in our particular case, education and entertainment.

Future experiments are needed to quantify the specific learning benefits of the Energy Surge System, but we hope that we have at least demonstrated that there is value in building service systems and using the case study method to help refine and possibly extend service science theory. One such extension is the addition of distributed cognition logic to guide the design of individual-level service systems.

In closing, Flyvbjerg [23] paraphrasing Kuhn notes that: "a scientific discipline without a large number of thoroughly executed case studies is a discipline without systematic production of exemplars, and a discipline without exemplars is an ineffective one." Case studies of service systems can serve as important catalysts for service science as a scientific revolution.

\section{Acknowledgments}

This material is based partly upon work supported by the National Science Foundation (NSF) under ECCS - 1231046. Any opinions, findings, and conclusions or recommendations expressed in this material are those of the author and do not necessarily reflect the views of the NSF.

\section{References}

[1] Bray, D., and Konsynski, B. "Virtual worlds: multidisciplinary research opportunities", ACM SIGMIS Database, 38, 2007: pp. 17-25

[2] Schroeder, R. "Defining virtual worlds and virtual environments", Journal of virtual worlds research, 1, 2008: pp. $1-2$

[3] Nardi, B. My life as a night elf priest: An anthropological account of World of Warcraft. University of Michigan Press, 2010 . 
[4] Boellstorff, T. Coming of age in Second Life: An anthropologist explores the virtually human. Princeton University Press, 2015.

[5] Bainbridge, W. "The scientific research potential of virtual worlds", Science, 317, 2007: pp. 472-476.

[6] Animesh, A., Pinsonneault, A., Yang, S.B. and Oh, W. "An odyssey into virtual worlds: exploring the impacts of technological and spatial environments on intention to purchase virtual products", MIS Quarterly, 35, 2011: pp. 789-810.

[7] Eschenbrenner, B., Nah, F. and Siau, K., "3-D Virtual Worlds in Education: Applications, Benefits, Issues, and Opportunities", Journal of database management, 19, 2008: pp. 91-110.

[8] Petrakou, A. "Interacting through avatars: Virtual worlds as a context for online education", Computers \& Education, 54, 2010: pp. 1020-1027.

[9] Chaturvedi, Alok R., Dolk, D., and Drnevich, P. "Design principles for virtual worlds", MIS Quarterly, 35, 2011: pp 673-684.

[10] Ducheneaut, N., Yee, N., Nickell, E. and Moore, R. "Building an MMO with mass appeal a look at gameplay in world of Warcraft", Games and Culture, 1, 2006: pp.281317.

[11] Maglio, P., \& Spohrer, J. (2008). Fundamentals of service science. Journal of the academy of marketing science, $36,18-20$.

[12] Vargo, S., \& Akaka, M. (2009). Service-dominant logic as a foundation for service science: clarifications. Service science, 1, 32-41.
[13] Vargo, S., \& Lusch, R. (2004). Evolving to a new dominant logic for marketing. Journal of marketing, 68, 1-17.

[14] Hutchins, E., Cognition in the wild. MIT Press, 1995.

[15] Norman, D. Things that make us smart. Basic Books, 1993.

[16] Hutchins, E. "How a cockpit remembers its speeds." Cognitive science, 19, 1995: pp. 265-288.

[17] Hollan, James, Edwin Hutchins, and David Kirsh. "Distributed cognition: toward a new foundation for humancomputer interaction research." ACM Transactions on Computer-Human Interaction (TOCHI), 7, 2000, 174-196.

[18] Connell, J. and Shafer, L. Structured rapid prototyping: an evolutionary approach to software development. Yourdon Press, 1989.

[19] Eisenhardt, K. "Building theories from case study research", Academy of management review, 14, 1989: pp. 532-550.

[20] Yourdon, E, and L. Constantine. Structured design: Fundamentals of a discipline of computer program and systems design. Prentice-Hall, New Jersey, 1979.

[21] Simon, Herbert A. The sciences of the artificial (p. 162). MIT Press, Cambridge, MA, 1996.

[22] von Alan, R. Hevner, et al. "Design science in information systems research." MIS Quarterly, 28, 2004: 75105 .

[23] Flyvbjerg, B. "Five misunderstandings about case-study research", Qualitative inquiry, 12, 2006: pp.219-245. 